\title{
PEDOT:PSS interfaces stabilised using a PEGylated crosslinker yield improved conductivity and biocompatibility
}

Matteo Solazzo ${ }^{a, b}$, Ainur Zhussupbekovac, Katarzyna Krukiewicz ${ }^{\mathrm{d}, \mathrm{e}}$, Karsten Fleischer ${ }^{\mathrm{c}, \mathrm{f}}$, Manus J. Biggs ${ }^{\mathrm{d}, \mathrm{g}}$, Michael G. Monaghan ${ }^{\mathrm{a}, \mathrm{b}, \mathrm{h} *}$

The rapidly expanding fields of bioelectronics, and biological interfaces with electronic sensors and stimulators, is placing an increasing demand on candidate materials to serve as robust surfaces that are both biocompatible, stable and electroconductive. Amongst conductive polymers, poly(3,4ethylenedioxythiophene):poly(styrenesulfonate) (PEDOT:PSS) is a promising material in biomedical research due to its appropriate stability and high conductivity, however its intrinsic solubility requires a crosslinking process that can limit its conductivity and biocompatibility. Poly(ethylene glycol) is known to be a suitably anti-immunogenic moiety and its derivatives have been widely used for biomedical applications. In this study we investigate the application of poly(ethylene glycol) diglycidyl ether (PEGDE) as an effective crosslinker and conductive filler for PEDOT:PSS. From our interpretation of XPS analysis we hypothesise that the crosslinking reaction is occurring via the epoxy ring of PEGDE interacting with the sulfonic groups of excel PSS chains, which reaches a saturation at 3\% PEGDE concentration. PEGDE crosslinked films did not disperse in aqueous environments, had enhanced electrical conductivity and imparted a significant degree of hydrophilicity to PEDOT:PSS films. This hydrophilicity and the presence of biocompatible PEGDE led to good cell viability and a significantly increased degree of cell spreading on PEDOT:PSS films. In comparison to widely reported chemical crosslinking via glycidoxy propyltrimethoxysilane (GOPS), this original crosslinking yields a highly hydrophilic $2 \mathrm{D}$ film substrate with increased electroconductive and biocompatibility properties, resulting in a nextgeneration formulation for bioengineering applications.

\section{Introduction}

The interface between electronics and biological components is a rapidly expanding field with applications within the next generation of implants and sensor devices, adaptive drug delivery systems ${ }^{1}$, and tissue engineered therapeutics. Such interfaces determine biocompatibility and the efficiency of the intended function. Since their initial discovery and landmark research towards the end of the last century ${ }^{2-4}$, conductive polymers (CPs) have gathered considerable attention due to several attractive features: tunability of physical properties, flexibility, stimuli responsiveness and processability ${ }^{5}$. Traditionally, CPs such as polyaniline (PANI) and polypyrrole (PPy) have been investigated and applied for several decades ${ }^{6}$, 7. However, their long-term stability and availability to be dispersed in non-toxic carriers or solvents to permit facile manipulation and fabrication through relatively easy, cheap and reliable techniques (i.e. spin coating, casting etc.) is lacking. Poly(3,4-ethylenedioxythiophene) (PEDOT) on the other hand, a conjugated polymer; is readily dispersed in aqueous solutions and can be doped with polystyrene sulfonate (PEDOT:PSS), to formulate a conducting polymer for biological applications with good conductivity, chemical stability and processability 8 . PEDOT:PSS possesses both ionic and electronic conductive properties and has been the subject of extensive research in the fields of microelectronics ${ }^{9}$, sensor technology ${ }^{10}$, actuation 11 and has been explored extensively in biological scaffold development, neural implant ${ }^{12}$ and optoelectronic applications 13 .

Previous reports have demonstrated PEDOT:PSS as cytocompatible in vitro ${ }^{14}$; however, crosslinking of PEDOT:PSS is often essential in order to prevent delamination and redispersion of conducting PEDOT:PSS films placed in aqueous physiological conditions for long-term applications. Efforts have explored methods such as UV light ${ }^{15}$, poly (ethylene oxide) treatment ${ }^{16}$ and in particular the use of silanes ${ }^{17}$; with notable attention focused towards glycidoxy propyltrimethoxysilane (GOPS) crosslinking of PEDOT:PSS 2D films ${ }^{18}$ and 3D scaffolds

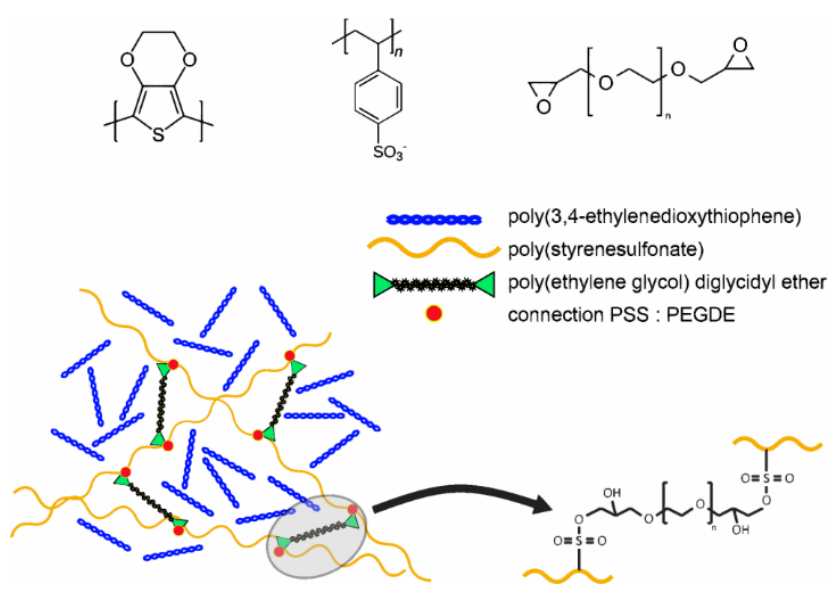

Scheme 1. At the top, from left to right: monomer of 3,4-ethylenedioxythiophene (EDOT), monomer of styrene sulfonate (SS) and poly (ethylene glycol) diglycidyl ether (PEGDE). At the bottom: hypothesized crosslinking reaction of PEGDE with PEDOT:PSS. The schematic demonstrates the proposed mechanism of epoxy rings at each end of the PEGDE reacting with SO3- moiety of PSS chains.

19. Such GOPS crosslinking is based on a hypothesis that the epoxy ring group in GOPS reacts with sulfonic groups of PSS chains leading to a change in PEDOT:PSS film morphology while the oxidation level of PEDOT remains unaffected ${ }^{18}$.

One disadvantage of GOPS crosslinking is that it requires high curing temperatures ( $140{ }^{\circ} \mathrm{C}$ for $\sim 1$ hour) and imparts a compromising effect on PEDOT conductivity values 20,21 . This also hinders incorporation of natural biomolecules during the fabrication process which can degrade at high temperatures. As a result of this, GOPS is often used in combination with secondary doping agents such as ethylene glycol or DMSO to restore some degree of conductivity ${ }^{22}$. As such, improved conductivity imparted by the addition of ethylene glycol has been attributed to a phase segregation of excess PSS resulting in the formation of a three-dimensional conducting network ${ }^{23}$. Considering this, we hypothesize that an alternative crosslinker, that simultaneously combines the conductive filler properties of ethylene glycol and an epoxy ring structure to bind with sulfonic groups present in excess PSS, can achieve superior crosslinking 
to generate stable structures whilst also achieving increased conductivity of PEDOT:PSS. Poly(ethylene glycol) diglycidyl ether (PEGDE) is a water soluble epoxy resin, considered a flexible chain polymer and has been adopted as a biocompatible crosslinker for freeze-dried silk sericin 24 and for low temperature processed silk fibroin films ${ }^{25,26}$. PEGDE molecules also have the potential to covalently bind with amino- and carboxyl groups of proteins as well as with hydroxyl groups via its epoxide terminations, and has been adopted as a crosslinker agent in redox hydrogels for biosensors ${ }^{27}$.

In this work we have employed PEGDE as an alternative crosslinker of PEDOT:PSS to enhance its stability for long-term biological applications and to allow resultant thin films to be cured at ambient temperatures. Suspensions of crosslinked material were optimised and characterized using $x$-ray Photoelectron Spectroscopy (XPS) and Fourier Transform Infrared Spectroscopy (FT-IR) to verify the reaction of crosslinking; with GOPS as a control. On physical assessment of crosslinked films, they resist dispersion in aqueous environments, are highly hydrophilic, and possess superior conductivity when compared to that of GOPS crosslinked films. We also demonstrate an enhanced biocompatibility and increased cell spreading on PEGDE crosslinked PEDOT:PSS films.

\section{Experimental Section}

\section{Preparation of crosslinked films}

PEDOT:PSS 1.3 wt.\% dispersion in water (Sigma-Aldrich, Ireland) was mixed with PEGDE (Sigma-Aldrich, Ireland) at concentrations of $0,1,3,5,10 \mathrm{w} / \mathrm{v} \%$. GOPS was employed at a concentration of $3 \mathrm{v} / \mathrm{v} \%$ as previously reported by others 19 . Solutions were vortexed for 30 seconds, sonicated for 30 minutes and filtered with a $0.45 \mu \mathrm{m}$ PVDF syringe filter to remove aggregates. Indium tin oxide (ITO) coated glass slides (Sigma-Aldrich, Ireland) were prepared by sonication in consecutive washes of soap (Liquinox, Alconox, Inc, USA), tetrahydrofuran, isopropanol: acetone 2:1 solution, isopropanol and distilled water. Slides were then dried in oven at $110^{\circ} \mathrm{C}$ for 20 minutes and surface activated by oxygen plasma treatment (Diener Electronic, Plasma Surface Technology, PICO, Germany) at $200 \mathrm{~W}$ for 10 minutes. Afterwards, $150 \mu \mathrm{l}$ of the filtered PEDOT:PSS blends were spin coated on the ITO surfaces at $1500 \mathrm{rpm}$ for 30 seconds using a spin coater (WS-400BX6nPP/LITE, Laurell, Technologies Corporation, Pennsylvania, USA), and dried to remove excess water. Films used for the physicochemical evaluation of crosslinking were left overnight at $37^{\circ} \mathrm{C}$; while substrates for the other tests were dried in oven at $120^{\circ} \mathrm{C}$ for 1 hour, as a higher drying temperature resulted in quicker evaporation and more homogeneous films. Thicker $2 \mathrm{D}$ films were prepared by drop casting $1 \mathrm{ml}$ of a mixture upon a metal substrate and evaporating the water content at room temperature overnight. PEDOT:PSS crosslinked controls (GOPS) were cured for an additional hour in oven at $140{ }^{\circ} \mathrm{C}$ based on established protocols ${ }^{18}$.

Assessment of Crosslinking: XPS, FT-IR, dispersion study
XPS was performed in an Omicron MultiProbe XPS using a monochromised Al $\mathrm{K} \alpha$ source (XM 1000, 1486.7eV). The instruments base pressure was $5 \times 10^{-11} \mathrm{mbar}$ and the instrumental resolution was $0.6 \mathrm{eV}$. To minimize unintentional surface contaminations all samples were prepared, cut and mounted in a fume hood and transported to the instrument in an inert atmosphere $\left(\mathrm{N}_{2}\right)$. Air exposure was minimised to a brief exposure of 30 seconds -1 minute during loading of the samples into the XPS. Spectra were analysed and fitted with CasaXPS (http://www.casaxps.com). Of particular interest is the fine structure of the sulphur (S) $2 p$ XPS peak as it is able to distinguish $S$ within the PSS and PEDOT subunits, and is sensitive to the chemical environment of the $\mathrm{SO}^{3-}$ groups in the PSS ${ }^{18,28}$. To analyse the $S 2 p$ we used multiple $S 2 p_{3 / 2}, S 2 p_{1 / 2}$ doublets with a fixed spin orbit split of $1.202 \mathrm{eV}$ and a line shape described by a sum of a Gaussian and Lorentzian peak (SGL, 10\% Gauss). Both splitting and shape had been previously determined on highly ordered, epitaxial $\mathrm{MoS}_{2}$ layers measured in the same instrument ${ }^{29}$. To describe the asymmetric line shape of the PEDOT related sulphur component with a minimum number of additional fitting parameters, an exponential asymmetric blend of the SGL shape was used. To quantitatively describe the peak changes for an increased cross-linker concentration all shape related fitting parameters were kept unchanged after optimisation for the plain PEDOT:PSS reference film (SGL ratio, Asymmetry parameter $T$ for the PEDOT component), while amplitude, peak positions and broadening of all components where fitted for each sample.

To further assess the crosslinking effectiveness of the substrate, the chemical composition of 2D drop-casted films was investigated with FT-IR using a PerkinElmer ${ }^{\circledR}$ Spectrum $100^{\mathrm{TM}}$ (PerkinElmer, USA) machine measuring 32 frames per sample. Afterwards, spectra were analysed with PerkinElmer ${ }^{\circledR}$ Spectrum software (PerkinElmer, USA) and characteristic peaks were identified.

Finally, macroscopic assessment of PEDOT:PSS crosslinking was performed by immersing 2D drop-casted films into distilled water. Specimens were incubated in petri dishes containing distilled water and sealed using parafilm at room temperature. Dynamic conditioning (100 rpm on orbital shaker) was applied for 48 hours, and images were obtained immediately before immersion in water and 48 hours later.

\section{Electroconductivity Characterization}

Electrochemical performance of PEDOT:PSS films was measured using a PARSTAT 2273 potentiostat in a three-electrode set-up, comprising a PEDOT-coated ITO glass slide as a working electrode, $\mathrm{Ag} / \mathrm{AgCl}(3 \mathrm{M} \mathrm{KCl})(\mathrm{EDAQ})$ as a reference electrode and a Ti/Pt rod (EDAQ) as an auxiliary electrode. Volumetric capacitance $\left(\mathrm{C}_{\mathrm{vol}}\right)$ was assessed basing on the cyclic voltammetric (CV) curves collected in a physiologically relevant $1 x$ PBS solution, within the potential range from -0.3 to $1.0 \mathrm{~V}$ (vs. $\mathrm{Ag} / \mathrm{AgCl}$ ) at $100 \mathrm{mV} \mathrm{s}^{-1}$ for $3 \mathrm{CV}$ cycles. $\mathrm{C}_{\text {vol }}$ values were calculated as the electric charge integrated under a corresponding CV curve during one CV cycle, divided by the volume of the specimen. The volumetric charge injection capacities $\left(\mathrm{ClC}_{\mathrm{vol}}\right)$ were quantified by the integration of 
chronoamperometric curves comprising a single biphasic potential pulse consisting of a $5 \mathrm{~ms}$ application of a reduction potential $(-0.5 \mathrm{~V}$ vs. $\mathrm{Ag} / \mathrm{AgCl})$ followed by a $5 \mathrm{~ms}$ application of an oxidative potential $(0.5 \mathrm{~V}$ vs. $\mathrm{Ag} / \mathrm{AgCl})$. Electrochemical Impedance Spectroscopy (EIS) spectra were collected in a $1 \mathrm{x}$ PBS solution within a frequency range from $100 \mathrm{mHz}$ to $100 \mathrm{kHz}$, with $\mathrm{AC}$ amplitude of $40 \mathrm{mV}$ (vs. $\mathrm{Ag} / \mathrm{AgCl}$ ) and $\mathrm{DC}$ potential equal to $0 \mathrm{~V}(v s$. $\mathrm{Ag} / \mathrm{AgCl})$. The results were presented on Bode plots and compared to those of a bare ITO electrode. EIS Spectrum Analyzer 1.0 software and the Powell algorithm were used to fit the experimental data to an equivalent circuit model. The simulations of the EIS data was performed with the use of an equivalent circuit model, comprising a combination of solution resistance $\left(R_{1}\right)$, charge transfer resistance $\left(R_{2}\right)$, two constant phase elements ( $C P E_{1}$ and $\left.C P E_{2}\right)$ and Warburg diffusion element (W), In order to ensure the high goodness of fit, the relative deviation of the calculated spectrum from the measured data was restricted not to exceed $2 \%$.

\section{Surface characterization}

Films were observed via brightfield microscope and their thicknesses were measured using a profilometer (Dektak 6M stylus profiler, Veeco, USA), providing also an estimation of roughness as arithmetical mean deviation Ra. Hydrophilicity measurements were performed with a FTA125 contact angle analyser (First Ten Angstroms, USA), by depositing a single droplet of distilled water onto the samples and measuring the angle between the surface and water droplet using the associated software. Tissue culture plastic $\mathrm{Ibidi}^{\circledR}$ petri dishes have been used as controls (Ibidi, Germany) (TCP).

\section{Cytotoxicity}

C3H10 mouse embryonic fibroblasts (ATCC ${ }^{\circledR}$ CCL-226TM) were cultured in growth media prepared with Dulbecco's Modified Eagle's Medium (DMEM) low glucose (Sigma-Aldrich) containing $10 \%$ foetal bovine serum (FBS) (Gibco ${ }^{\circledR}$ by Life Technologies) and 2\% Penicillin Streptomycin (Pen-Strep) (Sigma Aldrich) at $37 \stackrel{\circ}{\circ}$ with $5 \%$ CO2. Film substrates, at different crosslinker concentrations were prepared on ITO coated square glass slides with an approximate size of $10 \mathrm{~mm}$ per side and investigated for cytocompatibility. Substrates were then sterilised with multiple washes in $70 \% \mathrm{v} / \mathrm{v}$ ethanol solution and sterile deionized water, followed by UV for 30 minutes, and finally incubated in the growth media for 24 hours. Following the pre-conditioning, cells were seeded with a density of 2,000
(A)

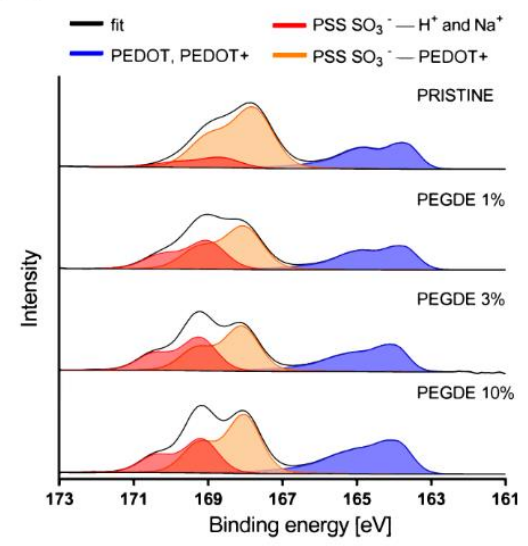

(E)

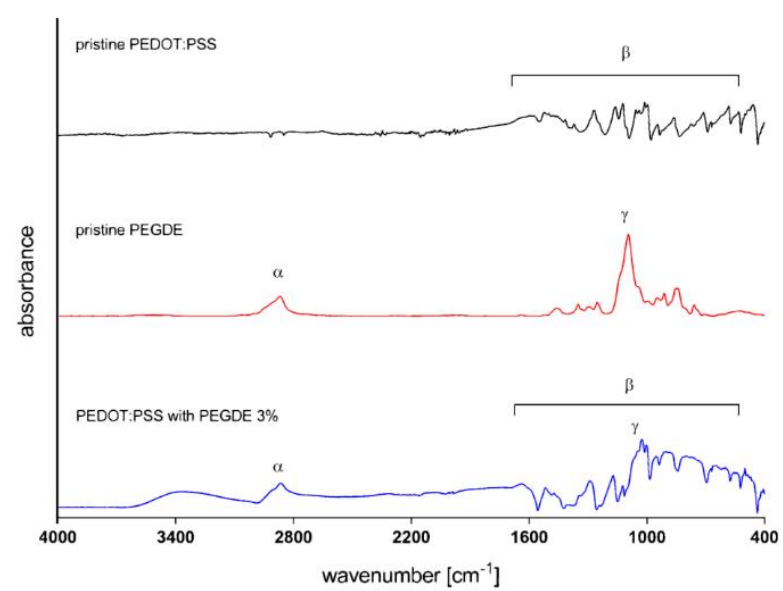

(B)

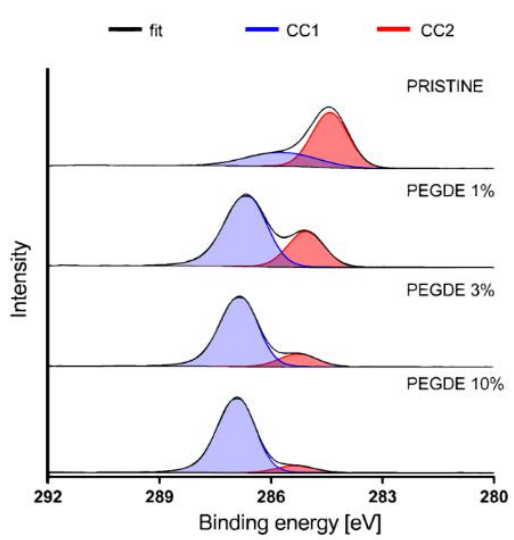

(F)
(C)

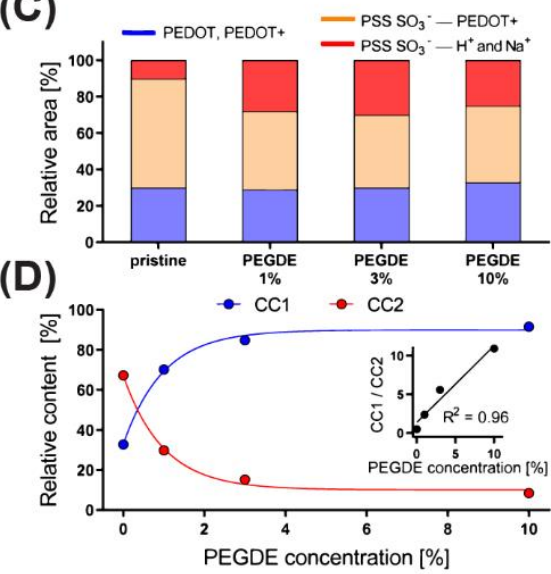

Figure 1. Establishment of PEDOT:PSS crosslinking using PEGDE. (A-D) XPS analysis on PEDOT:PSS samples with increasing PEGDE concentration. Spectra of (A) S2p and of (B) C1s core levels obtained on pristine and PEGDE crosslinked PEDOT:PSS films. (C) Relative areas of the three deconvolutions of S2p core levels and (D) the relative contents of the two deconvolutions of C1s core levels. (E) FT-IR spectra of pure PEDOT:PSS, PEGDE and PEDOT:PSS film crosslinked with PEGDE with characteristic peaks $\alpha, \gamma$ and band $\beta$. (F) Investigation of water dissolution of crosslinked and pristine PEDOT:PSS drop-casted films up to 48 hours. Scale bars: $10 \mathrm{~mm}$.

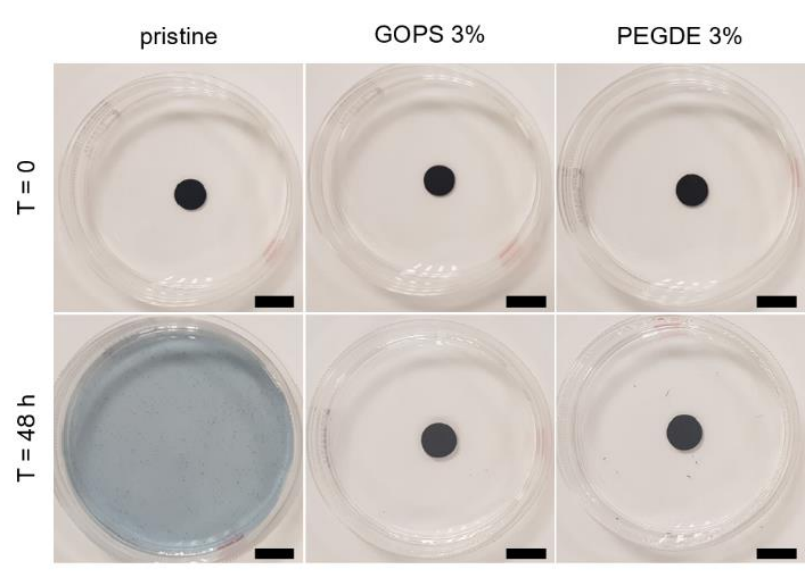


cells $/ \mathrm{cm}^{2}$ into the well containing film substrates with $\mathrm{Ibidi}^{\circledR}$ petri dishes as controls.

Following 48 hours incubation, the substrates were imaged using brightfield microscope, after which cell viability was assessed using a Live and Dead assay implementing a solution of $2 \mu \mathrm{l} / \mathrm{ml}$ Ethidium Homodimer and $0.5 \mu \mathrm{l} / \mathrm{ml}$ Calcein (Cambridge Bioscience) in PBS. Specimens $(n=4)$ were observed with a Leica SP8 scanning confocal microscope (Leica Microsystems, Germany), 3 pictures per experimental replicate were subsequently analysed with Image (freely available from www.nih.gov) and the cell viability was defined as live (green cells) as a proportion of the total cell number. Cell spreading was evaluated using cytofluorescent staining. Briefly, $n=3$ samples were fixed in $4 \% \mathrm{w} / \mathrm{v}$ paraformaldehyde for 60 minutes at room temperature, followed by incubation in a working dye solution of $1 \mu \mathrm{l} / \mathrm{ml}$ phalloidin (Santa Cruz Technology, USA) and 4',6-Diamidine-2'-phenylindoledihydrochloride (DAPI, $1 \mathrm{mg} / \mathrm{ml}$, Sigma-Aldrich, Ireland), to highlight filamentous actin (f-actin) of the cell cytoskeleton and cell nuclei respectively. Micrographs were obtained using a confocal microscope described above. Mean cell area was computed as the area of the picture positively stained for $f$-actin normalized by the number of cells.

\section{Statistical Analysis}

Statistical analysis was performed using GraphPad Prism 8 (GraphPad Software, USA). Where appropriate a one-way analysis of variance (ANOVA) followed by Tukey's multiple comparison. If not otherwise specified, results are presented as mean \pm standard deviation and differences are considered as statistically significant for $\mathrm{p}<0.05$.

\section{Results and discussion}

\section{PEGDE effectively crosslinks PEDOT:PSS at low temperature}

Chemical crosslinking of PEDOT:PSS has been mainly achieved via GOPS with high temperatures for annealing. Other works have alluded to the possibility of low temperature crosslinking via the use of divinylsulfone in blend but these also contain GOPS (albeit in minimal amounts) ${ }^{30}$. The use of a low temperature methods paves the way to new fascinating prospective of hybrid compound combining synthetic conductive polymers with natural proteins as decellularized biological material.

To verify our hypothesis concerning the interaction between PEDOT:PSS and PEGDE, XPS was performed on pristine and PEGDE crosslinked PEDOT:PSS films deposited on ITO coated glass slides (Figure 1). The spectra of S2p core levels (Figure 1.A) revealed that no sulphur was removed in the reaction, with the ratio between sulphur in PEDOT and in PSS blocks remaining constant for all crosslinker concentrations. Pristine PEDOT:PSS is $\mathrm{Na}$ doped ${ }^{31}$; possessing two PSS related components, namely the plain $\mathrm{SO}_{3}{ }^{-}$and the other the $\mathrm{SO}_{3}-\mathrm{X}$, where $\mathrm{X}$ can be $\mathrm{Na}$ or $\mathrm{H}$ atoms. The introduction of PEGDE does not influence the low binding energy band (163-167 eV) characteristic of C-S-C bonds within PEDOT chains. However, the high binding energy band (167-171 eV) proper of the $\mathrm{SO}_{3}^{-}$group in PSS chain was significantly affected by PEGDE, yielding a significant increase in reacted $\mathrm{SO}_{3}-\mathrm{X}$ groups represented by an increase in the deconvolution area attributed to this bond (Figure 1.C) and a shift of the peak up to $0.4 \mathrm{eV}$ to higher binding energy. These reactions saturate at low crosslinker content, and exponential fits of the data suggest that $96 \%$ of all possible bonds have occurred at $2 \mathrm{w} / \mathrm{v} \%$. The XPS on the Carbon (Figure 1.B) can be deconvoluted into two $\mathrm{C}$ components for all sample, and the higher binding energy component, indicated as the CC1 deconvolution, linearly increases (Figure 1.D and inset) with crosslinker concentration.

These findings strongly support an interaction between PEGDE and PSS, while PEGDE does not directly react with the PEDOT moieties. Specifically, we put forward that the crosslinking takes place between the epoxy ring groups of PEGDE molecules and 
(A)
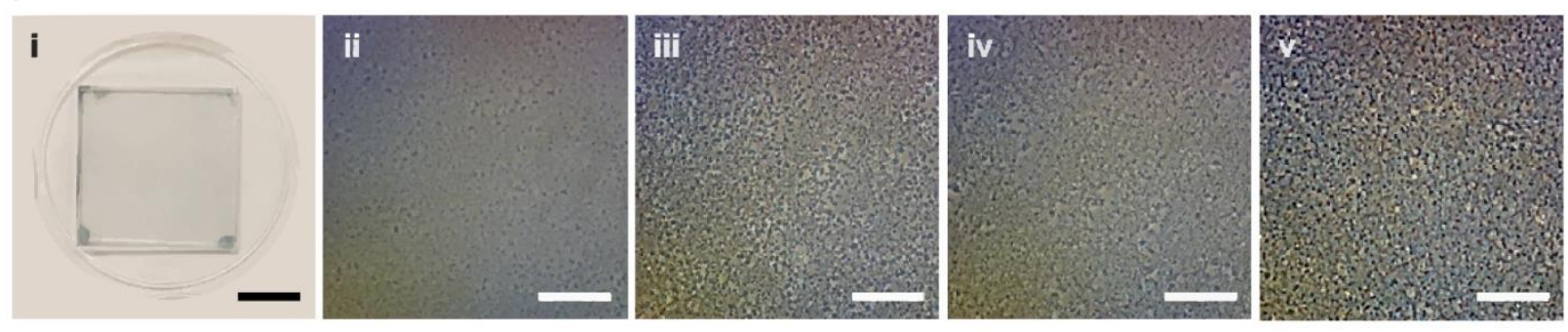

(B)

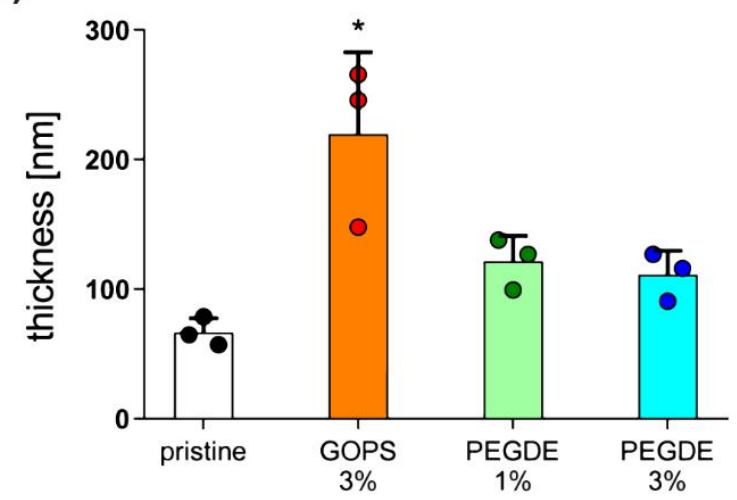

(C)

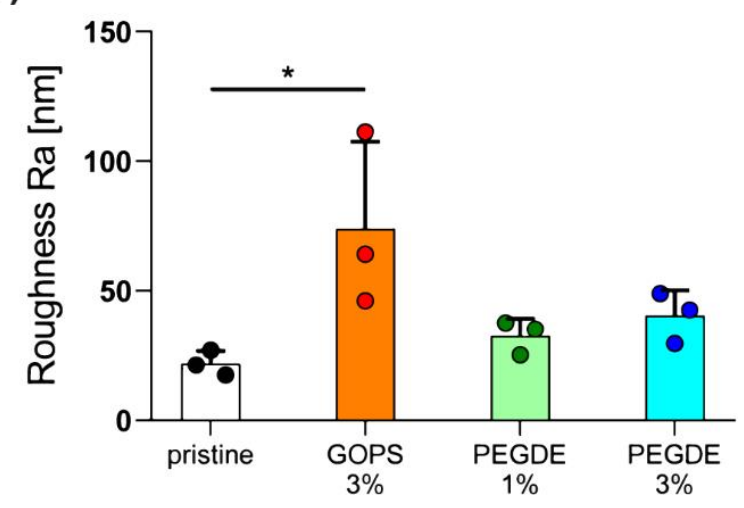

Figure 2. Profilometry characterization of crosslinked PEDOT:PSS films. (A) $i=$ picture of spin coated film on ITO, ii = pristine PEDOT:PSS, iii $=$ GOPS $3 \%$ crosslinked PEDOT:PSS, iv = PEGDE $1 \%$ crosslinked PEDOT:PSS, $v=$ PEGDE $3 \%$ crosslinked PEDOT:PSS. (B) Thickness quantification via profilometry. (C) Roughness quantification as arithmetical mean deviation Ra via profilometry. Scale bars: $A i=1 \mathrm{~cm}$, Aii-Av $=50 \mu \mathrm{m}$. Bar graphs demonstrate the mean of $\mathrm{n}=3$ with error bars representing standard deviation. Data values are presented as associated points. * represents statistical significance $(p<0.05)$ between indicated groups using one-way ANOVA with Tukey's post-hoc test.

the sulfonate groups of PSS; in a similar manner proposed for GOPS crosslinking of PEDOT:PSS by Hakånsson et al ${ }^{18}$. The shift to higher binding energy of the deconvolution $\mathrm{C} 1 \mathrm{~s}$ is index of a decrease in electron density that can be attributed to the bond between the delocalized electrons on PSS chain and the epoxy ring of $\mathrm{PEGDE}$. Moreover, the increase in the typical binding energy of $\mathrm{SO}_{3}-\mathrm{X}$ suggests a stronger interaction of the sulphur atoms of PSS with the PEGDE molecules than with the unaffected sulphur atoms of PEDOT molecules. Ultimately, the three relative areas of the deconvolutions of S2p core levels and (D) the relative contents of the two deconvolutions of C1s core levels confirm the reaction saturates at the concentration of 3 w/v\% PEGDE.

FT-IR spectra (Figure 1.E) of pure PEDOT:PSS, pure PEGDE and PEDOT:PSS scaffold crosslinked with $3 \mathrm{w} / \mathrm{v} \%$ PEGDE (respectively black, red and blue lines) have been obtained. The peaks $\alpha$ at $2867 \mathrm{~cm}^{-1}$ and $\gamma$ at $1094 \mathrm{~cm}^{-1}$ respectively characteristic of $-\mathrm{CH}$ stretch ${ }^{32}$ and ether bond ${ }^{33}$ of PEGDE, and the band $\beta$ between 600 and $1500 \mathrm{~cm}^{-1}$ of PEDOT:PSS ${ }^{34}$ are all present in the final structure, confirming the effective interaction of the two molecules. Further FTIR spectra, specifically the effect of increasing crosslinker content are reported in Supporting Information.1.

To investigate effective crosslinking at a macroscopic and physical level; drop-casted films were incubated for 48 hours in deionised water at room temperature under gentle rocking (Figure 1.F). Pristine PEDOT:PSS (first column) dispersed instantly with complete dissolution at 48 hours. PEGDE crosslinked samples (third column) did not disperse in aqueous conditions and exhibited similar stability to GOPS crosslinked samples (middle column). This result is of pivotal importance as it is evident confirmation of PEGDE efficiency as crosslinker with PEDOT:PSS to achieve a final compound able to stand dynamic condition at room temperature, and it entitled us to further proceed with studies in aqueous environment, such as cell culture.

\section{Crosslinking of PEDOT:PSS with PEGDE increases film conductivity}

It has been proven that the addition of plasticizers can enhance the ionic mobility and therefore increases the conductivity of a matrix ${ }^{35}$. Treating of PEDOT:PSS films with ethylene glycol to enhance conductivity has been extensively reported ${ }^{36,37}$. Its mechanism of improving conductivity has been attributed by Mengistie et al. to be through the selective removal of PSS which leads to an increase in thermoelectric performance of PEDOT:PSS ${ }^{38}$. Lin et al. have explored this further and concluded that the addition of ethylene glycol to PEDOT:PSS leads to the increase of the density of charge carriers and an increase in the mobility of these carriers with an overall modification of electron-phonon coupling ${ }^{39}$.

The evaluation of the electrochemical behaviour of all blends of PEDOT:PSS clearly showed the advantageous effects of using PEGDE as a crosslinking agent when compared with GOPS. As it can be seen in the EIS spectra (Figure 2A), PEDOT:PSS crosslinked with PEGDE $3 \mathrm{w} / \mathrm{v} \%$ exhibited the lowest impedance profile in a full range of investigated frequencies, outperforming other PEDOT:PSS formulations. The simulations of the EIS data with the use of an equivalent circuit model showed that the conductivity of PEDOT:PSS crosslinked with PEGDE $3 \mathrm{w} / \mathrm{v} \%$ reached a value of $706 \pm 32 \mathrm{~S} / \mathrm{cm}$, greatly outperforming the 
(A)

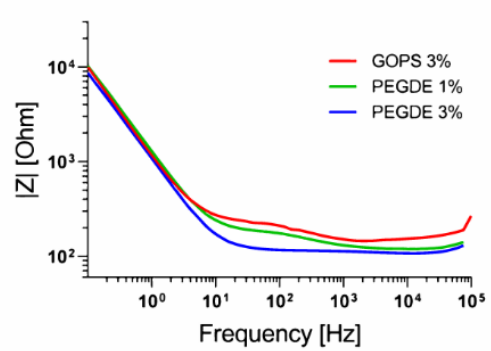

(E)

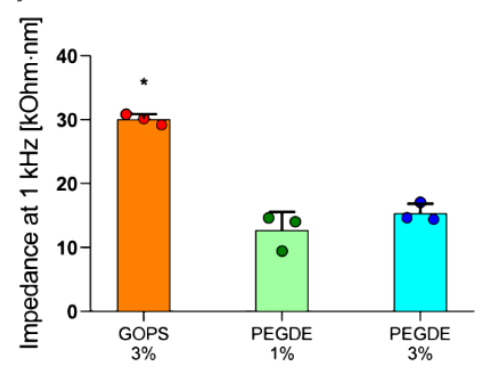

(B)

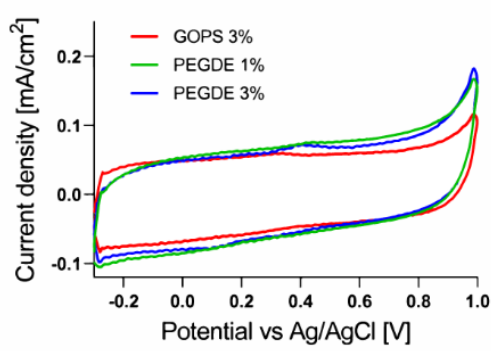

(D)

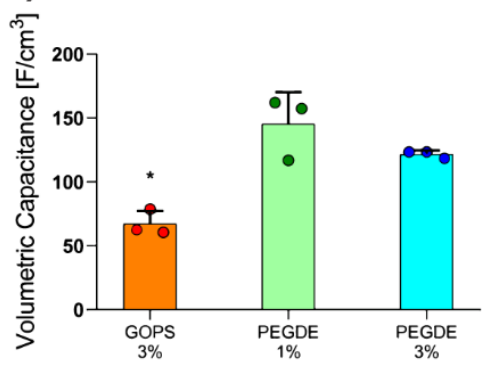

(C)

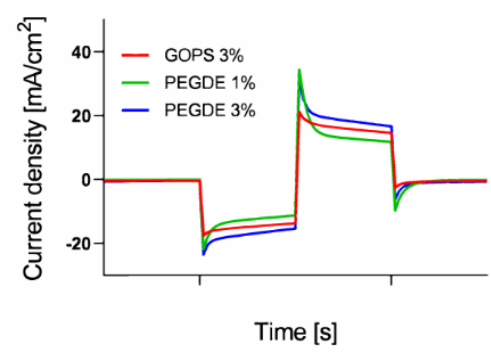

(F)

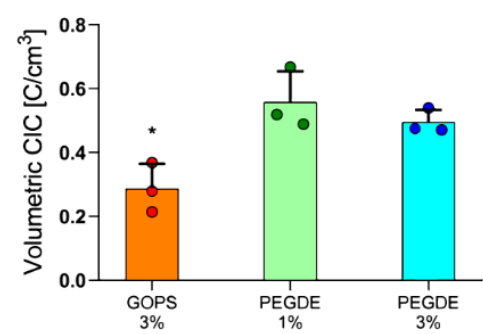

Figure 3. Electrochemical analysis of crosslinked PEDOT:PSS spin-coated on ITO coated glass slides. (A) Electrochemical Impedance Spectroscopy and (D) quantification of impedance at $1 \mathrm{kHz}$. (B) Cyclic Voltammetry and (E) quantification of Volumetric Capacitance ( $\mathrm{C}_{\mathrm{vol}}$ ). (C) Three pulse chronoamperometric testing and (F) quantification of Volumetric Charge Injection Capacitance $\left(\mathrm{CIC}_{\mathrm{vol}}\right)$. Data values are presented as associated points. * represents statistical significance $(\mathrm{p}<0.05)$ between indicated groups using one-way ANOVA with Tukey's post-hoc test.

conductivity of pristine PEDOT:PSS which is typically only $\sim 0.2$ $\mathrm{S} / \mathrm{cm}^{40}$. Furthermore, the low value of the impedance at $1 \mathrm{kHz}$ (Figure 2D), that was noted for both formulations of PEDOT:PSS crosslinked with PEGDE, indicated this chemistry as advantageous for neural stimulation/recording applications, since this frequency is biologically relevant and is used as the benchmark when analyzing the efficacy of electrode systems ${ }^{41}$. All reported CV curves (Figure 2.B) possessed a rectangular shape, which is typical for PEDOT in aqueous medium ${ }^{42}$ and indicates a capacitive behavior of these materials. Consequently, the calculation of charge passing through the electrode modified with PEGDE crosslinked PEDOT:PSS demonstrates that the use of PEGDE as the stabilizing agent led to a significant enhancement in the volumetric capacitance of PEDOT:PSS. Since both the calculations and previous experiments report the capacitance per volume of PEDOT:PSS to be typically of $6-57 \mathrm{~F} / \mathrm{cm}^{3}{ }^{43}$, the value of $121 \pm 2.9 \mathrm{~F} / \mathrm{cm}^{3}$ that was noted for PEDOT:PSS-PEGDE $(3 \% \mathrm{w} / \mathrm{v})$ clearly shows the efficiency of the described approach to enhance the electrochemical behavior of PEDOT:PSS. Although $2 \mathrm{C}_{\mathrm{vol}}$ describes the maximum amount of charge that can be stored within a polymer matrix in a steady state conditions, the actual biomedical applications usually employ short electrical pulses which are far from the equilibrium state. Therefore, it is the volumetric charge injection capacity $\left(\mathrm{ClC}_{\mathrm{vol}}\right)$ that indicates how much charge can be delivered in a single stimulation pulse. For instance, neural implants employ $\mathrm{CIC}$ values ranging from 2.3 $\mu \mathrm{C} / \mathrm{cm}^{2}$ to $2.3 \mathrm{mC} / \mathrm{cm}^{2} 44$. With a $\mathrm{ClC}_{\text {vol }}$ of approx. $500 \mathrm{mC} / \mathrm{cm}^{3}$, PEDOT:PSS-PEGDE can serve as an advantageous neural coating material capable of reaching a range of desired $\mathrm{CIC}$ values by tailoring its thickness.

\section{Crosslinking of PEDOT:PSS with PEGDE increases film hydrophilicity}

Spin coating yielded homogenous films (Figure 3.A) for all blends of PEDOT:PSS investigated, with the crosslinking formulations lending specific surface features. The typical configuration of PEDOT:PSS films consisting of rich PEDOT clusters in a lamellar backbone of PSS 45 was observable using brightfield microscope (Figure 3.A ii-v). A smooth coating with a

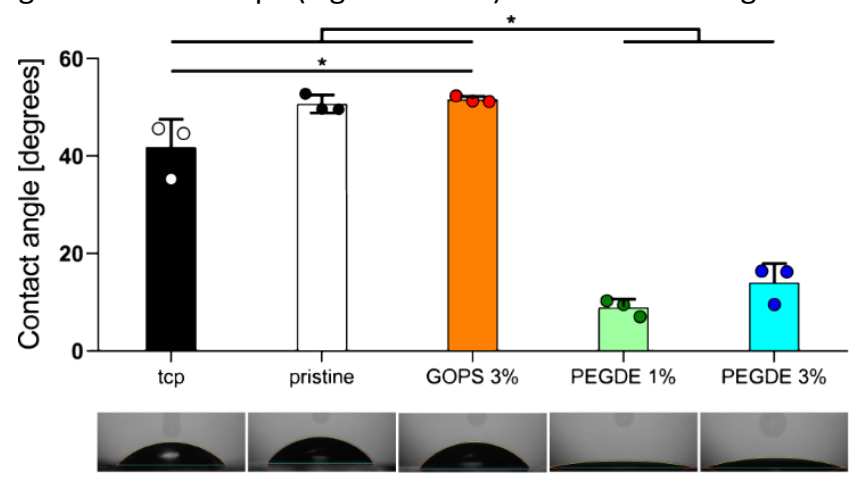

Figure 4. Hydrophilicity assessment via quantification of water contact angle measurement of spin coated films on ITO with tissue culture plastic Ibidi ${ }^{\circledR}$ petri dish (TCP) included as control. Bar graphs demonstrate the mean of $n=3$ with error bars representing standard deviation. Data values are presented as associated points. * represents statistical significance $(p<0.05)$ between indicated groups using one-way ANOVA with Tukey's post-hoc test.

low contrast between PEDOT rich and PSS rich sites was obtained with pristine PEDOT:PSS (Figure 3.A ii). Crosslinking of PEDOT:PSS conferred variable superficial features on resultant films with a high visual contrast present on GOPS $3 \mathrm{v} / \mathrm{v} \%$ (Figure 3.A iii) and PEGDE $3 \mathrm{w} / \mathrm{v} \%$ (Figure $3 . \mathrm{A} v$ ) crosslinked specimens, and to a lesser extent on PEGDE $1 \mathrm{w} / \mathrm{v} \%$ (Figure 3.A iv) crosslinked substrates. 
Quantification via profilometry confirmed that the GOPS crosslinked films were significantly thicker than those created using pristine PEDOT:PSS with or without PEGDE crosslinking. No significant increase in thickness occurred when PEGDE was introduced when compared to pristine PEDOT:PSS (Figure 3.B). Substrate thickness is an important characteristic of many materials utilized in biological applications as cells can sense the stiffness of underlying substrates at depths from $10 \mu \mathrm{m}$ onwards 46. However, the thicknesses obtained in our PEDOT:PSS films which were used for subsequent cell studies were all in the nanometre range (Figure 3B). Profilometry also enabled the estimation of film roughness as an arithmetical mean deviation Ra (Figure 3.C), and we found a similar trend to that of substrate thickness whereby Ra values were significantly higher on GOPS crosslinked films when compared with pristine PEDOT:PSS films. PEGDE crosslinked specimens did tend to yield Ra values higher than pristine PEDOT:PSS and lower than GOPS crosslinked samples, but these differences were not statistically significant. With regard to its application in biological sensors and materials, the role of surface roughness is not univocally defined; as authors have reported that a high roughness has been shown to increase surface adsorption of vitronectin and fibronectin, to achieve higher cell cardiomyocytes growth ${ }^{47}$, while others proved that increasing nanometer surface roughness enhanced the adhesion of vascular smooth muscle cell but decreased endothelial cell attachment ${ }^{48}$.

The use of PEG has been widely studied and reported in its ability to behave appropriately with blood components ${ }^{49}$, and it is used extensively to improve the biocompatibility of biomaterials for both in vivo and ex vivo applications ${ }^{50}$. This has been attributed to its resistance to protein adsorption which is important due to protein adsorption being a well-established first step in the response to foreign materials ${ }^{51}$. Given that the purpose of this study was to lend improved conductivity, stability and biocompatibility to PEDOT:PSS films using PEGDE; we also sought to determine the wettability of resultant films. Material hydrophilicity is known to have a reflection on cellular attachment and activity, and water contact angle investigation is a useful evaluation to predict cell interaction with the substrate ${ }^{52,53}$. Figure 4 highlights that the use of PEGDE crosslinking (at both $1 \mathrm{w} / \mathrm{v} \%$ and $3 \mathrm{w} / \mathrm{v} \%$ concentrations) significantly reduced the water contact angle and therefore increases the wettability and hydrophilicity of PEDOT:PSS films.

\section{PEDOT:PSS-PEGDE yields biocompatible films which increase cell spreading.}

CH310 mouse embryonic fibroblasts exhibited good cell viability on all samples and controls tested (Figure 5.A) with no significant difference amongst substrates and with all the values above $85 \%$ (Figure 5.B). Upon investigating cell morphology, it became strikingly apparent that a greater degree of cell spreading (determined via quantification of cell area) was occurring on PEDOT:PSS samples crosslinked using PEGDE (at both a $1 \mathrm{w} / \mathrm{v} \%$ and $3 \mathrm{w} / \mathrm{v} \%$ concentrations). Cell area was significantly increased on PEGDE $3 \mathrm{w} / \mathrm{v} \%$ crosslinked PEDOT:PSS samples when compared to GOPS $3 \mathrm{v} / \mathrm{v} \%$ crosslinked films and TCP as a control. Pristine PEDOT:PSS was not employed as a control in this study as it rapidly dissolved upon contact with cell culture media. Although cell area is higher on PEGDE $1 \mathrm{w} / \mathrm{v} \%$ crosslinked PEDOT:PSS scaffolds when compared to GOPS and TCP, no statistically significant increase is detected. The results of cell area possess a significant negative correlative relationship between surface contact-angle and cell area , with a Pearsons correlation coefficient $r=-0.85$ and $R^{2}=0.65$ (Figure 5.D). The increased cell spreading cannot be exclusively linked to the contact angle values reported in this study as there is a certain range of hydrophilicity deemed optimal for cell adherence and spreading ${ }^{53}$, outside of which is unfavourable for such cell behaviour. Indeed, the contact measurements reported in Figure 4 are outside of such limits ${ }^{54,55}$. Although surface roughness could come into play to the effect of cell spreading and adhesion, the surface roughness and magnitudes of variation between the groups are rather modest compared to those investigated in previous reports ${ }^{54}$. This leads us to conclude that the combined presentation of material chemistries, and preparation steps of PEDOT:PSS films for cell culture studies is driving this cell-spreading observation.

Previously reported mechanisms of PEDOT:PSS stabilisation have been previously proposed, such as the solution-processed crystallization reported by Kim et al. ${ }^{56}$. Similarly, crosslinking via PEGDE is a promising solution that may achieve thicker stable surfaces which could have great impact in the fields of electronics as charge storage systems or as more potent materials for bioelectronics. Moreover, PEGDE crosslinking of PEDOT:PSS does not require the use of toxic compounds or high temperature treatments, opening to the development of biohybrid constructs based on biological tissues. The beneficial integration of PEG in the PEDOT:PSS structure is not limited to just its effect on stabilisation, indeed it may confer appropriate biological properties, such as protein rejection, formation of two-phase systems with other polymers, and lack of immunogenicity and antigenicity ${ }^{57}$. Furthermore, the presence of the epoxy groups specific of the PEGDE molecule has shown to induce the adhesion of specific peptides 58 . We have demonstrated enhanced cell adhesion with no significant cytotoxicity as demonstrated by high cell viability. This present study focused employed a short-term in vitro study to evaluate the acute biocompatibility reaction and the material stability. Future work will embark on long-term studies together with the application of electrical stimulation 59 to access the potential of this new substrate to effectively deliver electrical stimulation and modulate tissue performances.

\section{Conclusions}

A novel PEGylated crosslinker to accomplish stable PEDOT:PSS substrates, which are highly stable and exhibit superior electrical conductivity, has been achieved. From XPS analysis we hypothesise that the crosslinking reaction is occurring via the epoxy ring of PEGDE interacting with the sulfonic groups of excel PSS chains, which reaches a saturation at 3\% PEGDE concentration. PEGDE crosslinked films did not disperse in aqueous environments, had enhanced electrical conductivity and imparted a significant degree of hydrophilicity to PEDOT:PSS films. This hydrophilicity and the presence of biocompatible PEGDE led to good cell viability and a significantly 
(A)

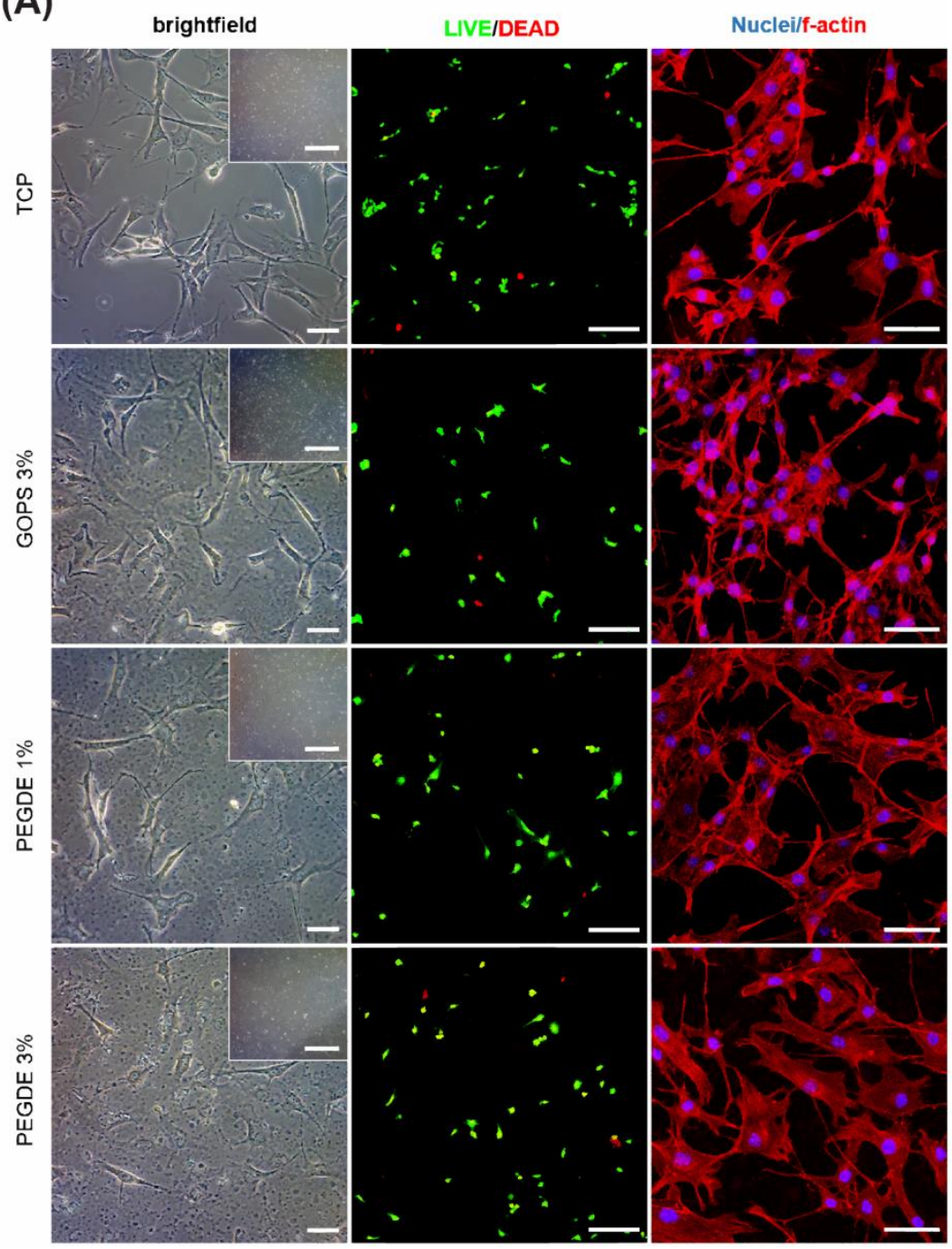

(B)

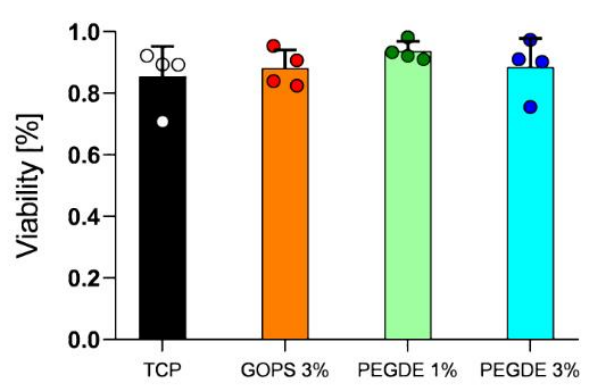

(C)

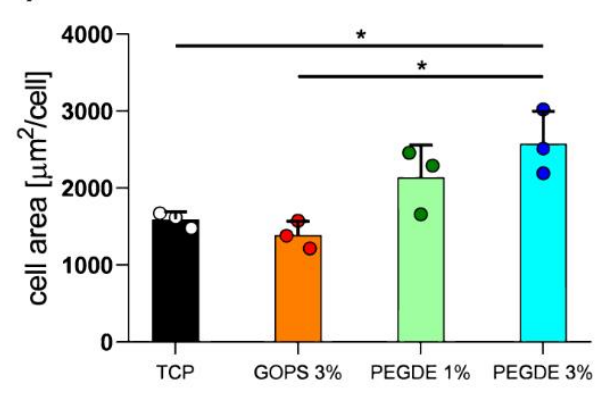

(D)

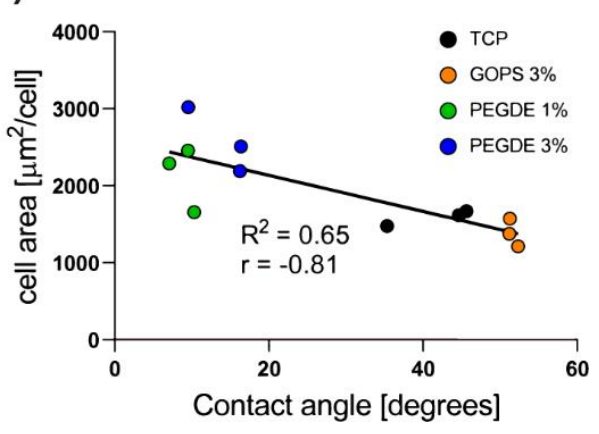

Figure 5. Cytocompatibility of crosslinked PEDOT:PSS films. (A) Micrographs of CH310 cells at 48 hours seeded on tissue culture plastic (TCP) and on PEDOT:PSS films crosslinked with GOPS 3 v/v\%, PEGDE $1 \mathrm{w} / \mathrm{v} \%$ and PEGDE $3 \mathrm{w} / \mathrm{v} \%$. First column and insets: brightfield microscope. Second and third columns showing confocal microscope fluorescent staining for live/dead and nuclei/f-actin respectively. (B) Quantification of viability (extracted from Live/Dead Staining) at 48 hours. (C) Quantification of cell surface area at 48 hours. (D) Pearsons correlation coefficient " $r$ " and $R^{2}$ analysis between contact angle and cell surface. Scale bars: brightfield $=50 \mu \mathrm{m}$, brightfield inset 500 $\mu \mathrm{m}$, Live/Dead $=200 \mu \mathrm{m}$, Nuclei/f-actin: $50 \mu \mathrm{m}$. Bar graphs demonstrate the mean of $\mathrm{n}=3$ with error bars representing standard deviation. Data values are presented as associated points. * represents statistical significance $(p<0.05)$ between indicated groups using one-way ANOVA with Tukey's post-hoc test.

increased degree of cell spreading on PEDOT:PSS films. This research has significant implications in the fields of biomaterials, sensor development and actuation whereby this molecule is serving both as a crosslinker and as facilitator of secondary dopancy. The opportunity to process these materials at room temperature at relatively physiological conditions offers the potential to incorporate natural biomolecules and proteins within PEDOT:PSS films for advanced biomaterial scaffolds. Such stability can facilitate the long-term measurement of electrical signals, the production of more advanced biomaterials and scaffolds for tissue engineering using a relatively straight-forward fabrication process.

\section{Conflicts of interest}

There are no conflicts to declare.

\section{Acknowledgements}

The authors wish to acknowledge Manuel Ruether, Department of Chemistry, TCD for assistance in FT-IR measurements, and Prof. Maria Santos-Martinez, School of Pharmacy and Pharmaceutical Sciences, TCD for advices on film processing. Ainur Zhussupbekova would like to acknowledge the support of the Kazakh government under the Bolashak program.

\section{References}

1. C. M. Proctor, A. Slézia, A. Kaszas, A. Ghestem, I. del Agua, A.-M. Pappa, C. Bernard, A. Williamson and G. G. Malliaras, Science advances, 2018, 4, eaau1291. 
2. A. J. Heeger, Angewandte Chemie (International ed. in English), 2001, 40, 2591-2611.

3. A. F. Diaz and J. A. Logan, Journal of Electroanalytical Chemistry and Interfacial Electrochemistry, 1980, 111, 111-114.

4. J. H. Burroughes, D. D. C. Bradley, A. R. Brown, R. N. Marks, K. Mackay, R. H. Friend, P. L. Burns and A. B. Holmes, Nature, 1990, 347, 539-541.

5. T. Nezakati, A. Seifalian, A. Tan and A. M. Seifalian, Chemical Reviews, 2018, 118, 6766-6843.

6. H. Shirakawa, E. J. Louis, A. G. MacDiarmid, C. K. Chiang and A. J. Heeger, Journal of the Chemical Society, Chemical Communications, 1977, DOI: 10.1039/C39770000578, 578-580.

7. A. Elschner, S. Kirchmeyer, W. Lovenich, U. Merker and K. Reuter, PEDOT: principles and applications of an intrinsically conductive polymer, CRC Press, 2010.

8. L. Groenendaal, F. Jonas, D. Freitag, H. Pielartzik and J. R. Reynolds, Advanced Materials, 2000, 12, 481-494.

9. P. Zhang, N. Aydemir, M. Alkaisi, D. E. Williams and J. TravasSejdic, ACS Appl Mater Interfaces, 2018, 10, 11888-11895.

10. I. Gualandi, M. Marzocchi, E. Scavetta, M. Calienni, A. Bonfiglio and B. Fraboni, Journal of Materials Chemistry B, 2015, 3, 67536762.

11. F. Wang, J.-H. Jeon, S. Park, C.-D. Kee, S.-J. Kim and I.-K. Oh, Soft Matter, 2016, 12, 246-254.

12. D. Khodagholy, T. Doublet, P. Quilichini, M. Gurfinkel, P. Leleux, A. Ghestem, E. Ismailova, T. Hervé, S. Sanaur, C. Bernard and G. G. Malliaras, Nature Communications, 2013, 4, 1575.

13. P. A. Levermore, R. Jin, X. Wang, L. Chen, D. D. C. Bradley and J. C. de Mello, Journal of Materials Chemistry, 2008, 18, 4414-4420.

14. S. Stritesky, A. Markova, J. Vitecek, E. Safarikova, M. Hrabal, L. Kubac, L. Kubala, M. Weiter and M. Vala, Journal of biomedical materials research. Part A, 2018, 106, 1121-1128.

15. L. Cao, Q. Tang and G. Wang, RSC Advances, 2016, 6, 2959229597.

16. T.-M. Huang, S. Batra, J. Hu, T. Miyoshi and M. Cakmak, Polymer, 2013, 54, 6455-6462.

17. S. Kim, A. Cho, S. Kim, W. Cho, M. H. Chung, F. S. Kim and J. H. Kim, RSC Advances, 2016, 6, 19280-19287.

18. A. Håkansson, S. Han, S. Wang, J. Lu, S. Braun, M. Fahlman, M. Berggren, X. Crispin and S. Fabiano, Journal of Polymer Science Part B: Polymer Physics, 2017, 55, 814-820.

19. A. G. Guex, J. L. Puetzer, A. Armgarth, E. Littmann, E. Stavrinidou, E. P. Giannelis, G. G. Malliaras and M. M. Stevens, Acta Biomaterialia, 2017, 62, 91-101.

20. S. Zhang, P. Kumar, A. S. Nouas, L. Fontaine, H. Tang and F. Cicoira, APL Materials, 2015, 3, 014911.

21. E. Stavrinidou, P. Leleux, H. Rajaona, D. Khodagholy, J. Rivnay, M. Lindau, S. Sanaur and G. G. Malliaras, Advanced Materials, 2013, 25, 4488-4493.

22. J. Nevrela, M. Micjan, M. Novota, S. Kovacova, M. Pavuk, P. Juhasz, J. Kovac Jr., J. Jakabovic and M. Weis, Journal of Polymer Science Part B: Polymer Physics, 2015, 53, 1139-1146.

23. X. Crispin, F. L. E. Jakobsson, A. Crispin, P. C. M. Grim, P. Andersson, A. Volodin, C. van Haesendonck, M. Van der Auweraer, W. R. Salaneck and M. Berggren, Chemistry of Materials, 2006, 18, 4354-4360.

24. W. Tao, M. Li and R. Xie, Macromolecular Materials and Engineering, 2005, 290, 188-194.

25. A. Motta, B. Barbato, C. Foss, P. Torricelli and C. Migliaresi, Journal of Bioactive and Compatible Polymers, 2011, 26, 130-143.

26. Y. Wei, D. Sun, H. Yi, H. Zhao and J. Wang, Journal of Wuhan University of Technology-Mater. Sci. Ed., 2014, 29, 1083-1089.

27. A. Heller and B. Feldman, Chemical Reviews, 2008, 108, 24822505.
28. M. M. de Kok, M. Buechel, S. I. E. Vulto, P. van de Weijer, E. A. Meulenkamp, S. H. P. M. de Winter, A. J. G. Mank, H. J. M. Vorstenbosch, C. H. L. Weijtens and V. van Elsbergen, physica status solidi (a), 2004, 201, 1342-1359.

29. F. O. V. Gomes, A. Pokle, M. Marinkovic, T. Balster, M. Canavan, K. Fleischer, R. Anselmann, V. Nicolosi and V. Wagner, Thin Solid Films, 2018, 645, 38-44.

30. D. Mantione, I. Del Agua, W. Schaafsma, M. ElMahmoudy, I. Uguz, A. Sanchez-Sanchez, H. Sardon, B. Castro, G. G. Malliaras and D. Mecerreyes, ACS Appl Mater Interfaces, 2017, 9, 1825418262.

31. G. Greczynski, T. Kugler, M. Keil, W. Osikowicz, M. Fahlman and W. R. Salaneck, Journal of Electron Spectroscopy and Related Phenomena, 2001, 121, 1-17.

32. S. Zheng, X. Li, Y. Zhang, Q. Xie, Y. S. Wong, W. Zheng and T. Chen, Int J Nanomedicine, 2012, 7, 3939-3949.

33. H. Li, Y. Lu and H. Liu, Journal of Composite Materials, 2016, 50, 1595-1602.

34. Q. Zhao, R. Jamal, L. Zhang, M. Wang and T. Abdiryim, Nanoscale research letters, 2014, 9, 557-557.

35. A. G. Supri, M. D. Siti Hajar and M. P. M. Hanif, Polymer Bulletin, 2016, 73, 2831-2841.

36. J. P. Thomas, L. Zhao, D. McGillivray and K. T. Leung, Journal of Materials Chemistry A, 2014, 2, 2383-2389.

37. J. Huang, P. F. Miller, J. S. Wilson, A. J. de Mello, J. C. de Mello and D. D. C. Bradley, Advanced Functional Materials, 2005, 15, 290 296.

38. D. A. Mengistie, C.-H. Chen, K. M. Boopathi, F. W. Pranoto, L.-J. Li and C.-W. Chu, ACS Applied Materials \& Interfaces, 2015, 7, 94100.

39. Y.-J. Lin, W.-S. Ni and J.-Y. Lee, Journal of Applied Physics, 2015, 117, 215501.

40. Z. Yu, Y. Xia, D. Du and J. Ouyang, ACS Applied Materials \& Interfaces, 2016, 8, 11629-11638.

41. K. Krukiewicz, M. Chudy, C. Vallejo-Giraldo, M. Skorupa, D. Wieclawska, R. Turczyn and M. Biggs, Biomed Mater, 2018, 13, 054102.

42. K. Krukiewicz, A. Kruk and R. Turczyn, Electrochimica Acta, 2018, 289, 218-227.

43. C. M. Proctor, J. Rivnay and G. G. Malliaras, Journal of Polymer Science Part B: Polymer Physics, 2016, 54, 1433-1436.

44. S. F. Cogan, Annual Review of Biomedical Engineering, 2008, 10, 275-309.

45. A. M. Nardes, M. Kemerink, R. A. J. Janssen, J. A. M. Bastiaansen, N. M. M. Kiggen, B. M. W. Langeveld, A. J. J. M. van Breemen and M. M. de Kok, Advanced Materials, 2007, 19, 1196-1200.

46. A. Buxboim, K. Rajagopal, A. E. Brown and D. E. Discher, Journal of physics. Condensed matter : an Institute of Physics journal, 2010, 22, 194116.

47. D. A. Stout, J. Yoo, A. N. Santiago-Miranda and T. J. Webster, International journal of nanomedicine, 2012, 7, 5653-5669.

48. D. C. Miller, A. Thapa, K. M. Haberstroh and T. J. Webster, Biomaterials, 2004, 25, 53-61.

49. B. C. Dash, G. Réthoré, M. Monaghan, K. Fitzgerald, W. Gallagher and A. Pandit, Biomaterials, 2010, 31, 8188-8197.

50. D. L. Elbert and J. A. Hubbell, Annual Review of Materials Science, 1996, 26, 365-394.

51. S. R. Sheth and D. Leckband, Proceedings of the National Academy of Sciences, 1997, 94, 8399-8404.

52. A. M. Pappa, V. Karagkiozaki, S. Krol, S. Kassavetis, D. Konstantinou, C. Pitsalidis, L. Tzounis, N. Pliatsikas and S. Logothetidis, Beilstein J Nanotechnol, 2015, 6, 254-262.

53. A. J. Ryan, C. J. Kearney, N. Shen, U. Khan, A. G. Kelly, C. Probst, E. Brauchle, S. Biccai, C. D. Garciarena, V. Vega-Mayoral, P. Loskill, 
S. W. Kerrigan, D. J. Kelly, K. Schenke-Layland, J. N. Coleman and F. J. O'Brien, Adv Mater, 2018, DOI: 10.1002/adma.201706442.

54. T. G. Ruardy, J. M. Schakenraad, H. C. van der Mei and H. J. Busscher, Journal of Biomedical Materials Research, 1995, 29, 1415-1423.

55. D. P. Dowling, I. S. Miller, M. Ardhaoui and W. M. Gallagher, Journal of Biomaterials Applications, 2011, 26, 327-347.

56. N. Kim, S. Kee, S. H. Lee, B. H. Lee, Y. H. Kahng, Y. R. Jo, B. J. Kim and K. Lee, Adv Mater, 2014, 26, 2268-2272, 2109.

57. J. M. Harris, Poly (ethylene glycol) chemistry: biotechnical and biomedical applications, Springer Science \& Business Media, 2013.

58. N. Vasylieva, B. Barnych, A. Meiller, C. Maucler, L. Pollegioni, J. S. Lin, D. Barbier and S. Marinesco, Biosens Bioelectron, 2011, 26, 3993-4000.

59. S.-M. Kim, N. Kim, Y. Kim, M.-S. Baik, M. Yoo, D. Kim, W.-J. Lee, D.-H. Kang, S. Kim, K. Lee and M.-H. Yoon, NPG Asia Materials, 2018, 10, 255-265. 